\title{
Chain Stability in Trading Networks
}

\author{
JOHN WILLIAM HATFIELD, University of Texas at Austin, USA \\ SCOTT DUKE KOMINERS, Harvard University, USA \\ ALEXANDRU NICHIFOR, University of Melbourne, Australia \\ MICHAEL OSTROVSKY, Stanford University, USA \\ ALEXANDER WESTKAMP, University of Cologne, Germany
}

We consider general trading networks with bilateral contracts. We show that a suitably adapted chain stability concept [4] is equivalent to stability [1,2] if all agents' preferences are jointly fully substitutable [3] and satisfy the Laws of Aggregate Supply and Demand (a condition we call monotone-substitutability). We also present three examples to show that are results are sharp, demonstrating that:

- If preferences of some agents do not satisfy the Laws of Aggregate Supply and Demand, then chain stable outcomes may not be stable.

- If preferences of some agents are not fully substitutable, then chain stable outcomes may likewise not be stable.

- If blocking sets are restricted to chains that do not "cross" themselves (i.e., chains that involve each agent in at most two contracts), then an outcome that is robust to such blocks may not be robust to richer blocks.

Our results imply that in trading networks with transferable utility, an outcome is consistent with competitive equilibrium if and only if it is not blocked by any chain of contracts.

We show moreover that, from a computational perspective, checking whether an outcome is chain stable is substantially easier than checking whether that outcome is stable directly. Indeed, we show that as the size of the economy grows, the number of chains of trades (corresponding to possible blocking chains) becomes infinitely smaller than the number of general sets of trades (corresponding to possible blocking sets).

Full Paper: https://ssrn.com/abstract $=3180740$

\begin{abstract}
We are grateful to Dave Donaldson, Alvin Roth, Alex Teytelboym, and Rakesh Vohra for helpful comments and suggestions. We thank Joseph Shayani for excellent research assistance. Kominers thanks the National Science Foundation (grants CCF-1216095 and SES-1459912, as well as a graduate research fellowship), the Harvard Milton Fund, the Yahoo! Key Scientific Challenges Program, the John M. Olin Center (a Terence M. Considine Fellowship), the Ng Fund and the Mathematics in Economics Research Fund of the Harvard Center of Mathematical Sciences and Applications, the American Mathematical Society, and the Simons Foundation for support. Nichifor received funding from the People Programme (Marie Curie Outgoing International Fellowship) of the European Union's Seventh Framework Programme (FP7/2007-2013) under REA grant agreement 625718, and thanks the Australian Research Council for financial support via a Discovery Early Career Researcher Award (DECRA). Ostrovsky thanks the Alfred P. Sloan Foundation for financial support. Westkamp received funding from the German Science Foundation and from the People Programme (Marie Curie Intra-European Fellowship) of the European Union's Seventh Framework Programme (FP7/2007-2013) under REA grant agreement 628276.
\end{abstract}

Authors' addresses: John William Hatfield, University of Texas at Austin, 2110 Speedway, B6600, Austin, TX, 78712, USA, john.hatfield@utexas.edu; Scott Duke Kominers, Harvard University, Rock Center 219, Harvard Business School, Boston, MA, 02163, USA, kominers@fas.harvard.edu; Alexandru Nichifor, University of Melbourne, 111 Barry Street, Carlton, Victoria, 3053, Australia, nichifor@unimelb.edu.au; Michael Ostrovsky, Stanford University, 655 Knight Way, Stanford, CA, 94305, USA, ostrovsky@stanford.edu; Alexander Westkamp, University of Cologne, Albertus-Magnus-Platz, 50923 Cologne, Germany,westkamp@wiso.uni-koeln.de.

Permission to make digital or hard copies of part or all of this work for personal or classroom use is granted without fee provided that copies are not made or distributed for profit or commercial advantage and that copies bear this notice and the full citation on the first page. Copyrights for third-party components of this work must be honored. For all other uses, contact the owner/author(s).

(C) 2018 Copyright held by the owner/author(s).

ACM EC'18, fune 18-22, 2018, Ithaca, NY, USA. ACM ISBN 978-1-4503-5829-3/18/06.

https://doi.org/10.1145/3219166.3219178 


\section{CCS Concepts: • Applied computing $\rightarrow$ Electronic commerce; Economics;}

Additional Key Words and Phrases: Matching, Stability, Networks, Substitutability

\section{REFERENCES}

[1] John William Hatfield and Scott Duke Kominers. 2012. Matching in Networks with Bilateral Contracts. American Economic fournal: Microeconomics 4 (2012), 176-208.

[2] John William Hatfield, Scott Duke Kominers, Alexandru Nichifor, Michael Ostrovsky, and Alexander Westkamp. 2013. Stability and Competitive Equilibrium in Trading Networks. Journal of Political Economy 121 (2013), 966-1005.

[3] John William Hatfield, Scott Duke Kominers, Alexandru Nichifor, Michael Ostrovsky, and Alexander Westkamp. 2018. Full Substitutability. (2018). Working Paper.

[4] Michael Ostrovsky. 2008. Stability in supply chain networks. American Economic Review 98 (2008), 897-923. 